\title{
Chest position and pulmonary deposition of surfactant in surfactant depleted rabbits
}

\author{
Roland Broadbent, Tai-Fai Fok, Myrna Dolovich, John Watts, Geoff Coates, \\ Barry Bowen, Haresh Kirpalani
}

\begin{abstract}
Aims-To investigate the correlation between chest position and the distribution of surfactant in the lungs of surfactant depleted rabbits, to corroborate current guidelines on the intratracheal instillation of exogenous surfactant in newborns.

Methods-Twelve tracheotomised rabbits, depleted of pulmonary surfactant by saline bronchoalveolar lavage, were given intratracheal 99m Technetium labelled Exosurf in three positions (prone, right side down, and left side down) $(n=4$ in each group). They were monitored for 10 minutes using dynamic gamma scintigraphy monitoring. Instillation completed, the lateral lying animals were turned to the opposite side to determine whether redistribution of the surfactant had taken place. The amount of radiolabelled surfactant deposited at the peripheral, central, dorsal and ventral parts of the lungs was then estimated by gamma counting of the lung sections at necropsy.
\end{abstract}

Results-Both gamma scintigraphy and gamma counting showed similar rates and total amount of surfactant accumulation in both lungs of the prone animals. In the lateral lying animals surfactant accumulated at a significantly faster rate in the dependent lungs: the amount of surfactant deposition was three to 14-fold that in the raised lungs $(p=0.017$; nested ANOVA). Changing the chest position immediately after instillation did not redistribute the surfactant. In all three groups of animals there was no significant difference in deposition between the peripheral, central, ventral and dorsal parts of the lungs.

Conclusions-Pulmonary distribution of intratracheally instilled surfactant is largely determined by gravity, and changing the chest position after instillation does not result in any redistribution of the surfactant. During the instillation of exogenous surfactant to newborn infants, keeping the chest in the horizontal position may therefore result in the most even distribution of the surfactant in the two lungs. Further deposition studies are required to evaluate the validity of the current recommendations on surfactant administration.

(Arch Dis Child 1995; 72: F84-F89)

Keywords: respiratory distress syndrome, surfactant, pharmacology, animal study.

There is evidence that for exogenous surfactant to be maximally effective in improving lung function, it should be uniformly distributed in the lungs. ${ }^{12}$ Previous studies have shown that both the volume of surfactant used $^{34}$ and the speed of administration ${ }^{5}$ have a significant effect on distribution. It has also been shown that if given prophylactically before the first breath, exogenous surfactant is more evenly distributed in the lungs of lambs than when it is given after establishment of ventilation. ${ }^{67}$ The effect of body position on the distribution of instilled surfactant is, however, unknown. The recommendations for positioning of infants during instillation vary from turning the infants to both sides, ${ }^{8}$ to giving a quarter of the surfactant while the infant is in each of four or five specific positions. ${ }^{9} 10$ Comparison of these two approaches, however, did not show any significant differences in the clinical outcomes of the infants. ${ }^{11}$ There have been claims that the distribution of surfactant is dependent on ventilation and that spreading phenomena, at least with natural surfactant, may facilitate delivery of surfactant to aerated lungs. ${ }^{12}$ As well as there being no information on the effect of position during instillation, we know nothing about the possibility of redistribution of surfactant with position changes after instillation.

Gamma camera scintigraphy has been used to monitor the dynamic accumulation of surfactant deposition ${ }^{12}$ but may have insufficient resolution to detect variations in surfactant distribution in small areas of the lungs. Static deposition has been assessed by radioactive counts of labelled surfactant in excised divided lungs. ${ }^{13}$ As each of these two modalities of investigation has its advantages we used both to study the effect of chest position during instillation on the initial distribution, and the subsequent redistribution on changing chest position.

The following describes our findings in a rabbit model of lung injury, using gamma 

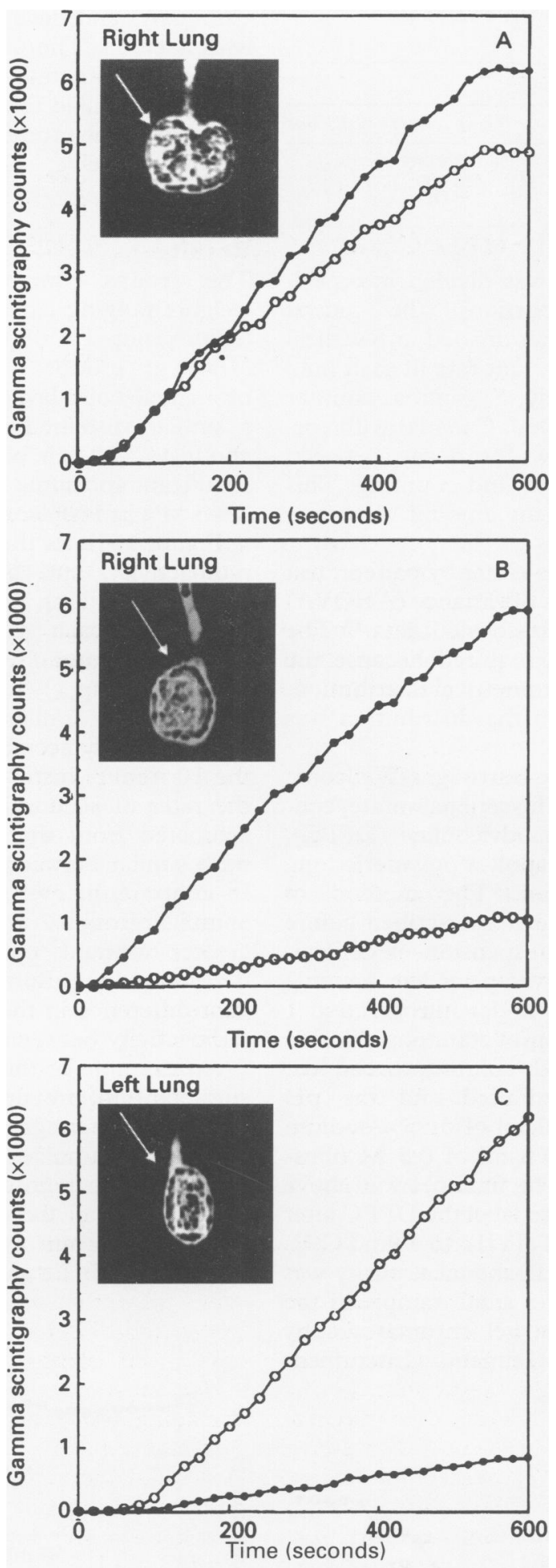

- Right lung $\circ$ Left lung

Figure 1 Example of dynamic lung scan from each of the three study groups taken during surfactant instillation.

(A) In prone (group 1) animals the rate of accumulation of radioactivity and the total amount deposited were similar in both lungs. The gamma camera image of the lungs and trachea (insert) also shows symmetrical distribution of radioactivity in both lungs. In contrast, radiolabelled surfactant accumulated at a faster rate and in greater amounts in the dependent lung of the laterally positioned animals lying either on the right (group 2) (B) or left (group 3) (C) side. The raised lung is hardly visible in the gamma camera image.

camera scintigraphy to examine the dynamic distribution of labelled surfactant, the effect of posture during delivery, and the effect of changing posture after instillation. This was combined with measurements of the absolute amount of surfactant in lung tissue specimens at necropsy from the same rabbit.

\section{Methods}

Twelve adult New Zealand white rabbits were depleted of surfactant using a bronchoalveolar lavage method based on that of Lachmann et al. ${ }^{14}$ The protocol was approved by the Animal Ethics Committee of McMaster University. The animals were anaesthetised using intramuscular acepromazine $(40 \mathrm{mg} / \mathrm{kg})$ and ketamine $(2.5 \mathrm{mg} / \mathrm{kg})$. A tracheostomy was performed and an intravenous line inserted to allow complete control of the airway and infusion of $10 \%$ dextrose $(10 \mathrm{ml} / \mathrm{hour})$ throughout the procedure. A tie was placed around the tracheostomy tube to prevent leakage of either air or surfactant.

The animal was then established on ventilation using a standard infant ventilator (Bournes BP 200) and paralysed using Pancuronium $100 \mathrm{mcg} / \mathrm{kg}$. The bronchoalveolar lavage was then performed with warm $\left(37^{\circ} \mathrm{C}\right)$ physiological saline $25 \mathrm{ml} / \mathrm{kg}$. The saline was run into the lungs at a hydrostatic pressure of $30 \mathrm{~cm}$ water, taking about 10 seconds for the infusion. Ten millilitres were then allowed to run out of the lungs and back in, following which the saline was drained from the lungs. Ventilation was started at a peak inflating pressure of $25 \mathrm{~cm}$ water, positive end expiratory pressure (PEEP) $4 \mathrm{~cm}$ water, rate $30 /$ minute, inspiratory time one second, and $100 \%$ oxygen. This procedure was performed four times with the animal supine and a further two times after turning the animal prone. The whole procedure took about 30 minutes.

After completion of the lavage, after about one hour for stabilisation, $99 \mathrm{~m}$ Technetium (99mTc) labelled surfactant (about 280 $\mu \mathrm{Ci} / \mathrm{ml})$, was instilled at a dose of $5 \mathrm{ml}(70$ $\mathrm{mg} / \mathrm{kg}$ down the endotracheal tube via the side port of the endotracheal tube connector supplied with the medication. The surfactant used was Exosurf (Burroughs Wellcome Laboratories, Canada). The instillation was carried out for 10 minutes at a constant rate with a syringe pump. During this time the distribution of $99 \mathrm{mTc}$ in the lungs was determined with an Ohio nuclear large field of view gamma camera interfaced to a digital PDP 11-23 computer. Consecutive five second frames were recorded for 10 minutes for a total of 120 frames.

There were three groups of animals, four in each group: group 1 prone lying; group 2 right side lying; and group 3 left side lying. For group 1 animals, continuous imaging on the gamma camera only was carried out throughout surfactant instillation. For groups 2 and 3, in addition to the continuous imaging during instillation, the rabbits were turned on to the other side immediately on completion of instillation, followed by dynamic imaging (120 five second images) for a further 10 minutes.

The animals were then given intravenous pentobarbitone and the lungs excised. To localise accurately the deposition, a predetermined necropsy procedure was followed: 
Table 1 Mean (SD) rate of accumulation of radioactivity in both lungs during instillation of surfactant

\begin{tabular}{lllll}
\hline \multirow{4}{*}{ Group } & \multicolumn{4}{l}{ Rate of accumulation of radioactivity (counts/second) } \\
\cline { 2 - 5 } & $n=$ & Right lung & Left lung & $p$ (paired t test) Value \\
\hline 1 Prone & 4 & $8 \cdot 61(1.35)$ & $8 \cdot 34(3.83)$ & 0.914 \\
2 Right side & 4 & $8 \cdot 99(1 \cdot 76)$ & $2 \cdot 07(0.82)$ & $0 \cdot 009$ \\
3 Left side & 4 & $1 \cdot 13(0 \cdot 32)$ & $9 \cdot 51(1.06)$ & 0.001 \\
\hline
\end{tabular}

each lobe of the lungs was divided into peripheral and central portions; the central portions were then further divided into ventral and dorsal pieces. The count rate in each lung piece was measured by a gamma counter (Minaxi auto-gamma 5000, Canada) with corrections made to allow for decay between manufacture of the tracer and counting. This allowed us to calculate the amount of surfactant in each lung piece.

Statistical analysis was either by paired $t$ test or nested design analysis of variance (ANOVA) on logarithmic (log) transformed data. ${ }^{15}$ The log transformation was required because the original data had an asymmetrical distribution. After log transformation the distribution was normal.

Exosurf Neonatal, the Burroughs-Wellcome proprietary name for colfosceril palmitate, contains dipalmitoylphosphatidylcholine (DPPC), cetyl alcohol, and tyloxapol, a polymeric long chain repeating alcohol. The method of labelling surfactant has been described before by Davis et al. ${ }^{12}$ The suspension of concentrated Exosurf $(3 \mathrm{ml})$ was placed in a sterile capped multidose phial under nitrogen and 1 $\mathrm{ml}$ of a $2 \mathrm{mg} / \mathrm{ml}$ solution of stannous chloride in $0.45 \%$ sodium chloride solution was added. The suspension was diluted and the $\mathrm{pH}$ adjusted by adding $13.2 \mathrm{ml}$ of $0.45 \%$ sodium chloride solution and $0.8 \mathrm{ml}$ of $0.5 \mathrm{M}$ phosphate buffer, $\mathrm{pH} 7 \cdot 6$. The final $\mathrm{pH}$ was above 6.5. The $99 \mathrm{mTc}$ seems to label the DPPC after reduction of the $99 \mathrm{mTc}(\mathrm{VII})$ to $99 \mathrm{mTC}(\mathrm{V})$ using stannous ion. Radiochemical purity was determined by spotting a small sample of the product on $7 \mathrm{~cm}$ silica gel chromatography strips (ITLC-SG Gelman Instrument

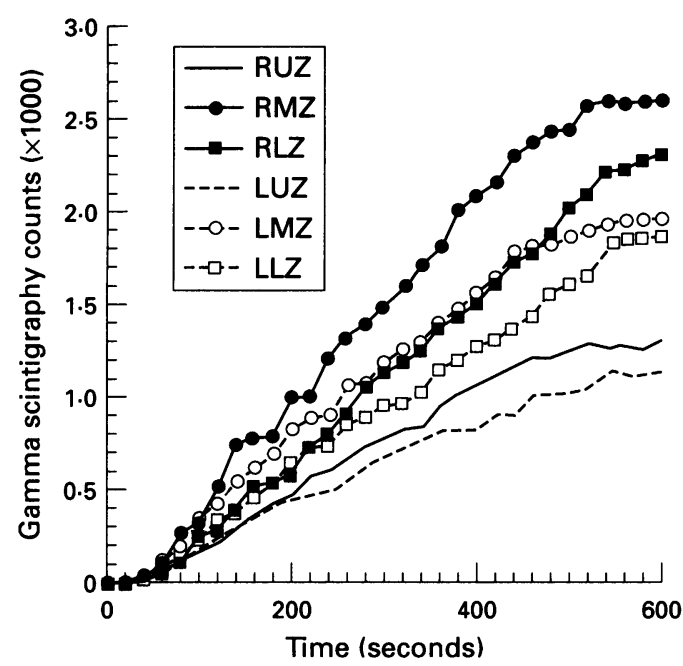

Figure 2 Dynamic scan on the accumulation of radiolabelled surfactant in the upper, middle, and lower regions of both lungs from a prone animal (group 1) during surfactant instillation.
Company) and developing the chromatogram with acetone. The unbound $99 \mathrm{mTc}$ travelled as the pertechnetate ion just behind the front while the labelled Exosurf- $99 \mathrm{mTc}$ remained at the origin. The product contained less than $5 \%$ unbound $99 \mathrm{mTc}$.

\section{Results}

The groups contained rabbits with body weights ranging from 2900 to $3250 \mathrm{~g}$ (mean (SD): group 1: 3080 (147) g; group 2: 3137 (160) g; group 3: 3050 (108) g). After bronchoalveolar lavage all rabbits experienced a similar sustained drop in tidal volume of about $40 \%$. Their blood gases also showed a significant and similar degree of hypoxia $\left(\mathrm{PaO}_{2}\right.$ $<9.3 \mathrm{kPa}$ in $100 \%$ oxygen).

Figure 1 shows the rate of accumulation of radioactivity, and hence surfactant, against time in each lung field of one representative rabbit from each group, as measured by the gamma scintigraphy. In all of the prone animals (group 1) (fig 1A) the gamma camera showed that similar amounts of surfactant were gradually accumulated in each lung over the 10 minute instillation. In the entire group the rates of accumulation of radioactivity, as estimated from the gradients of the curves, were similar between the right and left lungs. In contrast, in every one of the lateral lying animals (groups 2 and 3) (figs $1 B$ and 1C) a greater amount of surfactant entered the dependent lung. Both groups showed a significant difference in the rate of accumulation of radioactivity between the two lungs (table 1).

An example of the rate of accumulation of surfactant in the upper, middle, and lower regions of the lungs is illustrated in fig 2 . This dynamic accumulation was obtained from one of the animals in group 1. Filling occurs simultaneously in all three regions, but accumulation of surfactant was slowest in the upper zones of both lungs. This phenomenon was

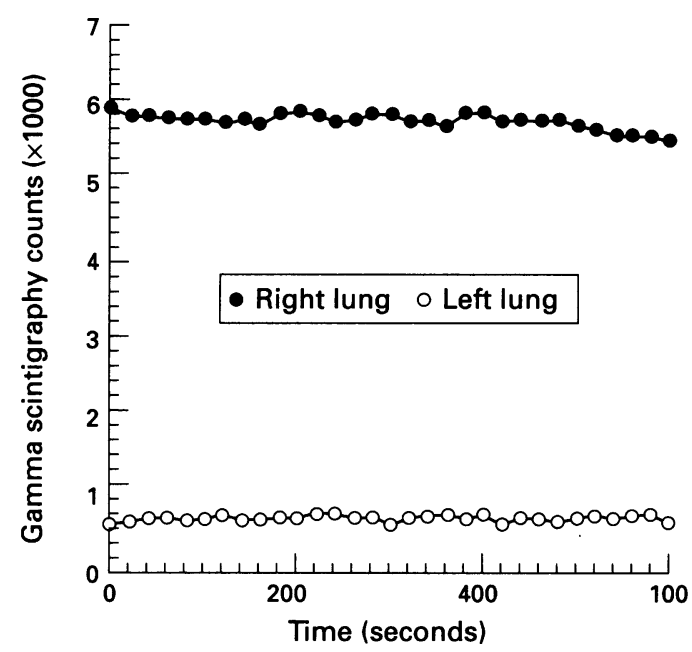

Figure 3 Dynamic scan taken after turning the rabbit to the opposite side on completion of surfactant instillation obtained from an animal which received the surfactant lying on the right side (group 2). There was no significant change in radioactivity counts in both lungs throughout the entire 10 minutes of the scan, showing that changing the chest position after completion of surfactant instillation is not followed by redistribution of the surfactant between the lungs. 
Table 2 Gamma camera counts (mean (SD)) in right and left lungs at 0 and 10 minutes after repositioning of lateral lying animals after instillation of surfactant

\begin{tabular}{lll}
\hline & \multicolumn{2}{l}{ Gamma camera counts } \\
\cline { 2 - 3 } Group & OMinute & 10 Minutes \\
\hline 2 Right side & $6636(1537)$ & $6535(1381)$ \\
$\begin{array}{l}\text { Right lung } \\
\text { Leff lung }\end{array}$ & $1138(613)$ & $1145(624)$ \\
3 Left side & $1106(273)$ & $1076(216)$ \\
$\begin{array}{l}\text { Right lung } \\
\text { Left lung }\end{array}$ & $6571(694)$ & $6327(686)$ \\
\hline
\end{tabular}

seen in both lungs in all three groups of animals.

Fig 3 shows a 10 minute dynamic scan taken from one animal in group 2 after it was turned on to its left side immediately following surfactant instillation given in the right side down position. There was no significant change in the amount of radioactivity in either lung after the animal was turned. This phenomenon was observed in all the eight lateral lying animals, showing that as soon as the instillation was completed, changing the chest position was not followed by any redistribution of the instilled surfactant between the lungs. The gamma camera counts in the right and left lungs at 0 minute and 10 minutes after repositioning of the lateral lying animals are shown in table 2. Analysis by paired $t$ test showed no significant difference $(p>0.05)$ in any of the paired values.

Fig 4 and table 3 show the amount of surfactant deposition in each lung, as estimated by counting the radioactivity in the lung

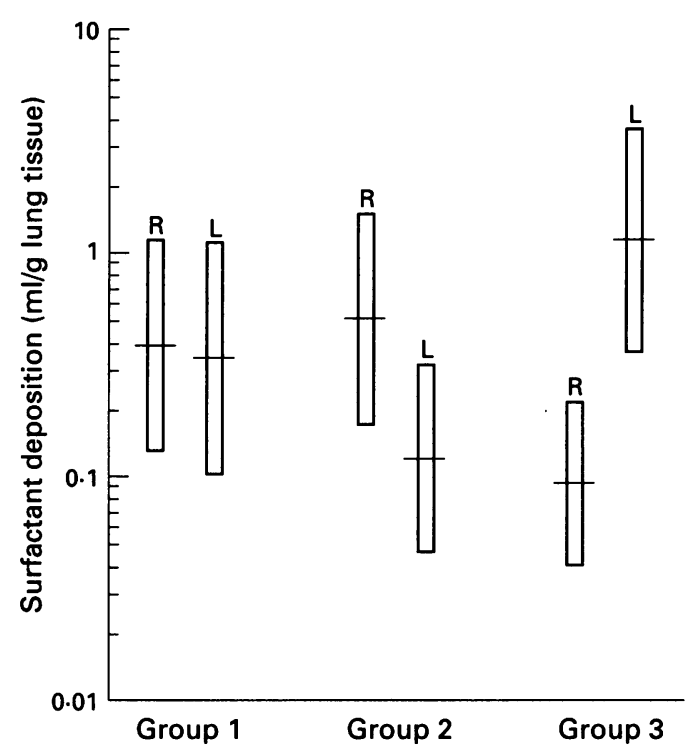

Figure 4 Geometric means (SDs) of the amount of surfactant deposited in the two lungs (expressed as $\mathrm{ml}$ of surfactant per gram lung tissue) in the three study groups. $R=$ right lung; $L=$ left lung.

Table 3 Geometric mean (SD) values of surfactant deposition ( $\mathrm{ml} / \mathrm{g}$ lung tissue) in right and left lungs in all three study groups

\begin{tabular}{lll}
\hline & \multicolumn{2}{l}{ Surfactant deposition ${ }^{\star}$} \\
\cline { 2 - 3 } Group & Right lung & Left lung \\
\hline 1 Prone & $0.388(+0.754,-0.256)$ & $0.340(+0.774,-0.236)$ \\
2 Right side & $0.507(+0.970,-0.333)$ & $0.121(+0.193,-0.074)$ \\
Left side & $0.095(+0.125,-0.054)$ & $1.145(+2.388,-0.774)$ \\
\hline *D &
\end{tabular}

*Difference in distribution pattern according to group: $p=0.017$, nested ANOVA. tissue with the gamma counter. In the prone lying animals (group 1) the amount of surfactant per gram of lung tissue is similar in the two lungs. However, in the lateral lying animals the dependent lungs contain more surfactant than the raised lungs. This difference in distribution pattern according to group was significant $(p=0.017$; nested ANOVA).

To study further the distribution of instilled surfactant within each lung each lobe of the lung was divided into peripheral and central portions. The central portions were also divided into ventral and dorsal sections. Thus a total of six lung tissue regions were available for comparison, three from each lung (peripheral, central dorsal, and central ventral). Fig 5 shows that regional distribution of surfactant in the two lungs was related to the chest position. The amount of surfactant per gram of lung tissue in every region was similar for both lungs in the prone lying animals. More surfactant was measured in every region of the dependent lung of the lateral lying animals. Data on the regional distribution of surfactant in both lungs from all three groups were combined to compare the deposition of surfactant in the peripheral, central dorsal, and central ventral regions (table 4). In all three groups the central parts of the lungs, especially the central ventral regions seemed to contain more surfactant per gram of lung tissue than the peripheral parts of the lungs. However, analysis by nested ANOVA did not reach significance.

\section{Discussion}

We postulated that there are three factors that could have a major influence on the pulmonary deposition of intratracheally instilled surfactant: (1) gravity, which would lead to deposition in the lower lung of a lateral lying animal; (2) ventilation, which would lead to deposition in the better ventilated lung; and (3) surfactant spreading phenomena. In this present experiment, we demonstrated the effect of gravity on the pulmonary deposition of surfactant. In the lateral lying animals the dependent lungs received significantly more surfactant than the raised lungs. In the prone animals, where the lungs were on the same horizontal plane, the surfactant was evenly distributed between the two lungs. We have also observed that changing the position of the lateral lying animals after completion of instillation of the radiolabelled surfactant did not result in any change in the radioactivity counts in the two lung fields. This showed that once deposited in the lungs exogenous surfactant does not redistribute with a further change of position. These findings strongly suggest that for the initial distribution of surfactant, gravity is the dominant factor. After initial distribution further redistribution, however, is not affected by changing the gravitationally dependent position.

Current clinical recommendations on the positioning of the infants during surfactant instillation of exogenous surfactant are varied. But, unfortunately, there are few published data to support the current practices. For example, it is recommended that infants be 


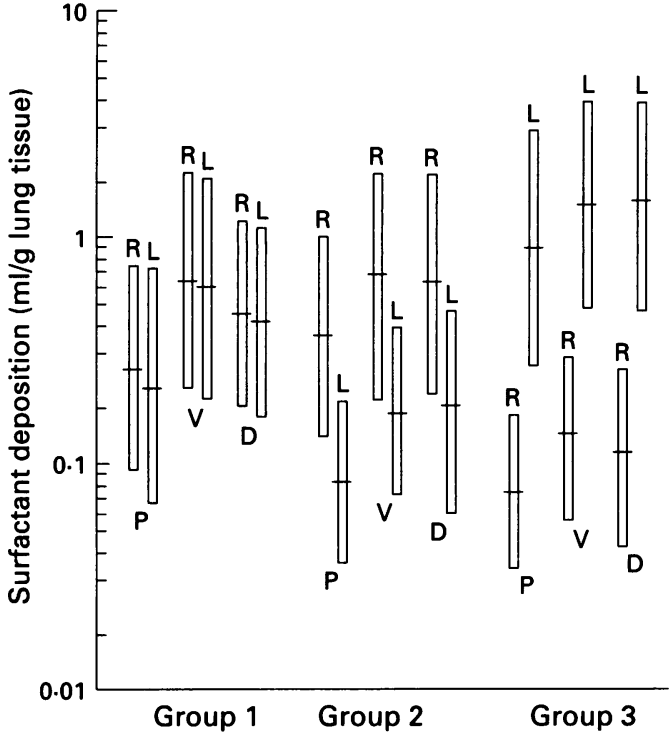

Figure 5 Geometric means (SDs) of the amount of surfactant deposited in each of the lung regions (expressed as ml of surfactant per gram lung tissue) in the three study groups. $P=$ peripheral, $V=$ ventral, $D=$ dorsal; $R=$ right lung, $L=$ left lung.

given Exosurf lying supine, but should be turned to lie on the left side and then the right side, each maintained for 30 seconds after completion of each half of instillation. ${ }^{8}$ This manoeuvre assumes that gravity will cause redistribution of surfactant between the two lungs after instillation. This, however, has been shown not to be the case in our animal model. The recommendations for the instillation of Survanta entail giving a quarter of the dose of surfactant while the infant is in each of four specific positions: head up and head down in each of the left lateral and right lateral positions. ${ }^{9}$ This manoeuvre, which is presumably intended to facilitate entry of the surfactant into the various lobes of the lungs, requires a considerable additional handling of the infants, including temporary disconnection from the ventilator. Theoretically, it may also increase the risk of maldistribution of surfactant as the lung regions that receive the surfactant first will become better aerated than the other parts of the lungs. In a multicentre randomised trial, Zola et al gave infants with respiratory distress syndrome Survanta through a catheter inserted into the endotracheal tube; the infants were positioned either by the 'Exosurf approach' or the 'Survanta approach.'11 There were no significant differences between the two approaches with regard to the infants' clinical outcomes (ventilation requirements, oxygenation, mortality and incidence of pulmonary airleaks) in the first 72 hours of life. ${ }^{11}$ However, this study did not include a group of infants who were given surfactant with the chest placed in the horizontal position.
Our experiment shows that when instilled with the animal lying in a horizontal position, exogenous surfactant is evenly distributed and accumulates at a similar rate in the two lungs. The rate of deposition in the upper, middle, and lower regions of the lungs seems also to follow the overall rate for the whole lung. There seems to be a tendency for the surfactant to concentrate at the central part of the lungs but this tendency is also seen in the animals lying laterally during surfactant administration. In both the prone lying and lateral lying animals there were no significant differences in deposition between ventral and dorsal parts of the lungs.

Our data, however, should be interpreted with caution when being applied to actual clinical practice. The distribution of surfactant may be affected by the anatomy of the bronchial tree which is not the same in rabbits and human infants. The animals in our study were kept in the prone rather than the supine position throughout the experiment because we found it easier to keep the chest in a straight horizontal position with the animals lying prone. In the actual clinical setting infants are usually placed in the supine position when surfactant is being administered, and we cannot assume that the surfactant behaves similarly in the lungs of prone and supine lying subjects. Recent evidence from animal experiments suggests that both homogeneous distribution of exogenous surfactant within the lungs and sustained improvement in oxygenation are achieved by fast bolus injection of the surfactant over a matter of seconds but not by slow infusion. ${ }^{5}$ There is also evidence that pulmonary distribution is more even if the surfactant is given in relatively large volumes. ${ }^{34}$ In our study a standard dose of surfactant was given to each animal by relatively slow infusion. It will be of interest to study whether the chest position will affect the distribution of surfactant given in fast split boluses or in larger volumes.

Despite their limitations our data cast some doubts on the validity of the current recommendations on intratracheal surfactant delivery. Further deposition studies with the animals being turned to the various positions, as recommended for Exosurf and Survanta, are required to determine the most appropriate chest position for surfactant administration. Unless the current practices are proved to be better, it may be desirable simply to position the infants in the horizontal position without any lateral tilt of the chest during surfactant instillation. This may eliminate possible hypoxia and cardiovascular disturbances associated with the handling of the infants using currently recommended instillation techniques.

This study was made possible by the generosity of Burroughs Wellcome Laboratories (Canada) who supplied the surfactant and who endowed a research fellowship for Dr Tai-Fai Fok.

Table 4 Geometric mean (SD) values of surfactant deposition (mVg lung tissue) in peripheral, central dorsal, and central ventral regions of lungs in all three study groups

\begin{tabular}{llll}
\hline Lung region & Group 1 & Group 2 & Group 3 \\
\hline Peripheral & $0 \cdot 240(+0 \cdot 490,-0 \cdot 161)$ & $0 \cdot 176(+0 \cdot 406,-0 \cdot 123)$ & $0 \cdot 263(+1 \cdot 045,-0 \cdot 210)$ \\
Central dorsal & $0 \cdot 440(+0 \cdot 676,-0 \cdot 267)$ & $0 \cdot 330(+0 \cdot 617,-0 \cdot 235)$ & $0 \cdot 380(+1 \cdot 558,-0 \cdot 306)$ \\
Central ventral & $0 \cdot 621(+1 \cdot 240,-0 \cdot 414)$ & $0 \cdot 341(+0 \cdot 765,-0 \cdot 236)$ & $0 \cdot 426(+1 \cdot 548,-0 \cdot 334)$ \\
\hline
\end{tabular}


We thank Ms Sheila Williams, biomedical statistician, University of Otago Medical School, Dunedin, New Zealand, for her statistical assistance.

1 Charon A, Taeusch HW, Fitzgibbon C, Smith GB, Treaves ST, Phelps DS. Factors associated with surfactant treatment response in infants with severe respiratory distress syndrome. Pediatrics 1989; 83: 348-54

2 Heldt GP, Merritt TA, Golembeski D, Gilliard N, Bloor C, Spragg R. Distribution of surfactant, lung compliance, and aeration of preterm rabbit lungs after surfactant therapy and conventional and high-frequency oscillatory ventilation. Pediatr Res 1992; 31: 270-5.

3 Gilliard N, Richman PM, Merritt TA, Spragg RG. Effect of volume and dose on the pulmonary distribution of exome and dose on the pulmonary distribution of exogenous surfactant administered to normal rabbits or to 1990; 141: 743-7.

4 van der Bleek J, Plotz FB, van Overbeek FM, Hiekimp A, Beehvis H, Widevuur ChRH, et al. Distribution of exogenous surfactant in rabbits with severe respiratory failure: the effect of volume. Pediatr Res 1993; 34: 154-8.

5 Segerer H, van Gelder W, Angenent FWM, van Woerkens LJPM, Cursteot T, Obleden M, et al. Pulmonary distribution and efficacy of exogenous surfactant in lung-lavaged rabbits are influenced by the instillation technique. Pediatr Res 1993; 34: 490-4.

6 Jobe A, Ikegami M, Jacobs H, Jones S. Surfactant and pulmonary blood flow distributions following treatment of premature lambs with natural surfactant. $\mathcal{f}$ Clin Invest 1984; 73: 848-56.

7 Walther FJ, Kuipers IM, Gidding CEM, Willebrand D, Buchholtz RTF, Bevers EM. A comparison of highfrequency oscillation superimposed onto backup mechanical ventilation and conventional ventilation on the distribution of exogenous surfactant in premature lambs. Pediatr Res 1987; 22: 725-9.

8 Long W, Thompson T, Sundell H, Schimacker R, Volberg F, Guthrie R, et al. Effects of two rescue doses of a synthetic surfactant on mortality rate and survival without bronchopulmonary dysplasia in 700 - to 1350-gram infants with respiratory distress syndrome. $\mathcal{F}$ Pediatr 1991 ; 118: 595-605.

9 Soll RF, Hoekstra RE, Fangman JJ, Corbet AJ, Adams JM James LS, et al. Multicentre trial of a single-dose modified bovine surfactant extract (Survanta) for prevention of respiratory distress syndrome. Pediatrics 1990; 85: 1092-102.

10 Fujiwara T, Konishi M, Chida S, Okuyama K, Ogawa $Y$, Takevchi Y, et al. Surfactant replacement therapy with a single postventilatory dose of reconstituted bovine surfactant in preterm neonates with respiratory distress syndrome: final analysis of a multicenter, double-blind drome: final analysis of a multicenter, double-blind, randomized trial and compar
Pediatrics 1990; 86: 753-64.

11 Zola EM, Gunkel JH, Chan RK, Lim MO, Know I Feldman BM, et al. Comparison of three dosing procedures for administration of bovine surfactant to neonate with respiratory distress syndrome. $\mathcal{F}$ Pediatr 1993; 122 453-9.

12 Davis JM, Russ GA, Dickerson B, Greenspan BS. Shor term distribution kinetics of intratracheally administered exogenous lung surfactant. Pediatr Res 1992; 31: 445-50.

13 Lewis JF, Ikegami M, Jobe AH, Tabor B. Aerosolized surfactant treatment of preterm lambs. $\mathcal{f}$ Appl Physiol 1991; 70: 869-76.

14 Lachmann B, Robertson B, Vogel J. In vivo lung lavage as an experimental model for the respiratory distress an experimental model for the respiratory distrome. Acta Anaesthesiol Scand 1980; 24: 231-6.

15 Winer BJ. Statistical principles in experimental design. New York: McGraw-Hill, 1962: 184-91. 